\title{
Short-Term Precipitation Forecasting Rolling Update Correction Technology Based on Optimal Fusion Correction
}

\author{
Meijin Huang1, Qing Lin1, Ning Pan1, Nengzhu Fan1, Tao Jiang1, Qianshan He², Lingguang Huang1 \\ ${ }^{1}$ Fujian Meteorological Observatory, Fuzhou, China \\ ${ }^{2}$ Shanghai Meteorological Service, Shanghai, China \\ Email:^oxeye75@163.com
}

How to cite this paper: Huang, M. J., Lin, Q., Pan, N., Fan, N. Z., Jiang, T., He, Q. S., \& Huang, L. G. (2019). Short-Term Precipitation Forecasting Rolling Update Correction Technology Based on Optimal Fusion Correction. Journal of Geoscience and Environment Protection, 7, 145-159. https://doi.org/10.4236/gep.2019.73008

Received: September 20, 2018

Accepted: March 25, 2019

Published: March 28, 2019

Copyright $\odot 2019$ by author(s) and Scientific Research Publishing Inc. This work is licensed under the Creative Commons Attribution International License (CC BY 4.0).

http://creativecommons.org/licenses/by/4.0/

\begin{abstract}
In order to improve the availability of regional model precipitation forecast, this project intends to use the measured heavy rainfall data of dense automatic stations to carry out historical precipitation in the high resolution: the Severe Weather Automatic Nowcast System (SWAN) quantitative precipitation forecast and the High-Resolution Rapid Refresh (HRRR) regional numerical model precipitation forecast in short-term nowcasting aging. Based on the error analysis, the grid fusion technology is used to establish the measured rainfall, HRRR regional model precipitation forecast, and optical flow radar quantitative precipitation forecast $(\mathrm{QPF})$ three-source fusion correction scheme, comprehensively integrate the revised forecasting effect, adjust the fusion correction parameters, establish an optimal correction plan, generate a frozen rolling update revised product based on measured dense data and short-term forecast, and put it into business operation, and perform real-time effect rolling test evaluation on the forecast product.
\end{abstract}

\section{Keywords}

Optimal Fusion Correction, Radar QPF, Numerical Model, Short-Term Precipitation Forecasting, Rolling Test

\section{Introduction}

The trend forecast of the development of severe weather system is the biggest difficulty of short-term forward forecasting. The model precipitation forecast becomes an important support for short-term precipitation forecasting business, but the model usually has "spin-up" problem (Long et al., 2014). Therefore, it affects the reliability of $1-6 \mathrm{~h}$ before the forecast. The Radar Quantitative Preci- 
pitation Forecast (QPF) has a small error before 2 - $3 \mathrm{~h}$, but for a rapidly developing and weakened storm system, the forecast error will increase rapidly with time. The fusion method not only considers the accuracy of the radar QPF forecast in predicting the location of the precipitation system, but also considers the ability of the numerical model forecast to reflect the changes of the precipitation system, avoiding the limitations of the single method (Dong, 2018; Zhang et al., 2017; Tanessong et al., 2017).

Because radar QPF and numerical precipitation forecast have their own strengths (Chen et al., 2004), through the fusion technology, using the measured rainfall data and high spatial-temporal resolution radar quantitative precipitation forecast QPF to scan the model precipitation forecast, can be greatly improved. The accuracy of model precipitation forecast effectively compensates for the current shortage of such products. Therefore, the accuracy of precipitation forecasting also makes it play a greater role in weather forecasting, especially short-term temporary forecasting, which helps to improve meteorological disaster prevention and mitigation. At the same time, it also further improves the pertinence and practicality of flood control weather services (Sakijege et al., 2014; Kissi et al., 2015; Huang et al., 2011).

\section{Data Selection and Data Preprocessing}

This paper selects the rainfall data of the automatic station in the dense area of Fujian Province, the SWAN radar reflectivity factor puzzle data (Fujian radar jigsaw network, radar model CINRAD/SA) and the reflectivity factor puzzle extrapolation forecast ZI relationship QPF data by China Meteorological Administration Provided by the Severe Weather Automatic Nowcast System (SWAN; Hu et al., 2011). The time resolution of the product output is $6 \mathrm{~min}$, the horizontal spatial resolution is $1 \mathrm{~km} \times 1 \mathrm{~km}$, the forecasting time of the extrapolated forecast data is $1 \mathrm{~h}$, and the horizontal spatial resolution is $3 \mathrm{~km} \times 3 \mathrm{~km}$ hourly WRF mode precipitation product.

Quality control of automatic station rainfall data (Awadallah \& Awadallah, 2013), using "fast quality control" automatic station minute level data, detecting minute level rainfall large value points and using radar data cross-check or QPE correction to eliminate singular points. Time matching of radar QPF and mode precipitation forecasting and intra-provincial radar time synchronization problem: By synthesizing radar QPF precipitation in 6 minutes, it can match the hourly HRRR regional numerical model precipitation time, and the 6 radar time synchronization in the province can be controlled in 2 minutes. Spatial matching of radar QPF and model precipitation prediction: using objective analysis methods such as Cressman (Goerss \& Jeffries, 1994) to downscale radar QPF.

\section{Fusion Method Introduction}

\subsection{Precipitation Forecast Error Test Evaluation}

Using the historical rainfall data, using historical statistical data analysis, the 
QPF of the SWAN, the optical flow method extrapolation forecast precipitation and the HRRR regional numerical model precipitation were respectively subjected to the precipitation TS score by 1 hour, 3 hours, and 6 hours in the immediate forecasting time. Equal point-to-point testing, as well as statistical analysis and evaluation of CSI index errors.

\subsubsection{QPF Product Inspection Method}

Pairing grid selection method for automatic station rainfall data and precipitation forecast: Firstly, the automatic station rainfall data and QPF data are tested according to the latitude and longitude consistency pairing test.

Specific inspection methods:

1) The closest method: select the grid point closest to the rainfall station as the center and expand to the surrounding $3 \times 3$ total of 9 grid points, take the grid point $\mathrm{QPF}$ value which is closest to the precipitation station live value, and check the error between the two; 2) 9-point averaging method: select the grid point closest to the rainfall station as the center and expand to the surrounding $3 \times 3$ total 9 grid points, take the 9 -point average QPF value and the rainfall station precipitation actual value, and check the error between the two; 3) The QPF of the nearest point of the whole point, the QPF of the 9-point averaging method, the corresponding hourly rainfall data, the rain station name, and the station number generation text file are generated one file per hour.

According to the test results of the 2015 Swan Radar Precipitation Forecast QPF, it is found that there are some differences in the results of different weather systems, system change speeds and echo movement speeds. For the echo motion, the large stable precipitation with long duration, the radar QPF error is smaller, and it is generally smaller than the actual situation. For the convective system with uneven distribution of local development, the QPF error is relatively large compared with the actual precipitation. For a precipitation system with less stable position and less change in echo intensity, the QPF error is not large. Overall, QPF products are concentrated between the evaluation factor $\mathrm{E}(\mathrm{S})$ of 0.5 1.4 and the error rate of $0.2-0.65$.

With the formula:

1) Evaluation factor:

$$
E(S)=\frac{\sum_{i=1}^{N} Q_{R i, t}}{\sum_{i=1}^{N} Q_{G i, t}}
$$

2) Error rate:

$$
F(S)=\frac{\sum_{i=1}^{N}\left|Q_{R i, t}-Q_{G i, t}\right|}{\sum_{i=1}^{N} Q_{G i, t}}
$$

where $N$ is the number of precipitation stations participating in the assessment throughout the region, $Q_{R i, t}$ and $Q_{G i, t}$ is the hourly cumulative 
amount measured by the radar QPF and the rain gauge at the $t$-th hour of the i-th rainfall station.

\subsection{Comparative Analysis of Optical Flow Method Extrapolation Forecast and Practical Application of SWAN Forecast Products}

Two convective process data were selected, and the radar combined reflectivity factor was extrapolated for 0 - 2 hours by optical flow method, and compared with SWAN products (i.e. cross-correlation method).

\subsubsection{Optical Flow Method Combined with Semi-Lagrangian Tracking Extrapolation Algorithm (Hereinafter Referred to as Optical Flow Method) Minute-Level Extrapolation Precipitation Prediction Method}

The optical flow method is used to track the motion of the echo. For the fast convective precipitation weather process with fast change, the optical flow method can capture the motion vector, so it has obvious forecasting advantages. Although the cross-correlation method is relatively ideal in a relatively flat stratified cloud precipitation system, the tracking failure in the strong convective weather will increase significantly, which will affect the final forecasting result. Cross-correlation method used in SWAN business systems. The optical flow method can make up for the defects of the traditional cross-correlation method, improve the extrapolation effect of the convective echo, improve the prediction ability of the radar echo movement, and improve the performance of the convective nowcasting system.

The semi-Lagrangian scheme extrapolates the echo, which maintains the rotation of the echo and improves the echo prediction. Due to the large vorticity in typhoon and strong weather, the single motion trajectory has a certain rotation effect. In order to solve the linear extrapolation without considering the shortcomings of rotation, the semi-Lagrangian advection scheme is used to extrapolate the radar echo.

\subsubsection{Comparative Analysis of Practical Applications}

1) Case study No. 1

On April 11, 2017, the strong convective weather process in Fujian was a general convective weather process. On the 11th, a strong convective weather distribution zone appeared in the south of Hunan to the central part of Fujian; scattered convective weather appeared over the south of Guangdong, and the border between Fujian and Guangdong There was also a strong convective weather. At 15 o'clock (Beijing time), the echoes entered Jiangxi from Jiangxi, and the strongest echo intensity was $40-45 \mathrm{dBz}$. The strong echo region above $30 \mathrm{dBz}$ exhibits a bow-shaped distribution (figure omitted), with a northerly wind intrusion on the back side and a southwesterly wind on the south side, which is generally a bow-shaped echo structure.

Using the optical flow method combined with the Lagrangian tracking extra- 
polation algorithm, the shape and position of the strong convection obtained by the extrapolation prediction (Figure 1(c)) are close to those observed by the radar (Figure 1(a)). The system has better predicted the echoes in central and southern Fujian and the strong echoes in western Fujian. Of course, comparing Figure 1(a) and Figure 1(c), it can be seen that the moving speed of the south segment of the echo is slow. Comparing the forecast and the actual situation, the optical flow method basically predicts the position of the maximum reflectivity factor characteristic. The strong centers on the live echoes are mainly distributed in the west, middle and south sections: the west section is mainly large-area echoes, and the strongest echoes are about $40 \mathrm{dBz}$. The middle and south sections are arcuate echoes, and the strongest echoes are about It is $50 \mathrm{dBz}$. The strong echo center after optical flow prediction is also mainly in the western, middle and southern sections. The strongest echo in the southern section is more south than the live, and the middle section is equivalent, but the maximum value is $50 \mathrm{dBz}$ larger than the live echo. From the comparison of the 1-hour forecast of this process and the echo situation after 1 hour, it can be seen that the strong echo position is consistent with the echo reality after 1 hour, and the shape is also close to the actual situation, while the SWAN product (Figure $1(b))$ is The echo positions are similar, and the mid-range strength is obviously weaker and northerly. Therefore, the optical flow method is also of great reference value for the one-hour extrapolation forecast of a large range of convective systems.

2) Case study No. 2

On April 24, 2017, the convective weather process in Fujian, on the 24th, there was a strong convective weather over the province. The intensity was relatively general. There were local convection processes in the province, which was less intense than the 11th. The main body of the storm was in Zhejiang. In our province, the echoes below $40 \mathrm{dBz}$ are dominant, and the shape and position of the strong convection obtained by the optical flow extrapolation prediction (Figure 2(c)) are close to those observed by the radar (Figure 2(a)). The SWAN product (Figure 2(b)) has a weaker intensity, and the area above $35 \mathrm{dBz}$ is less

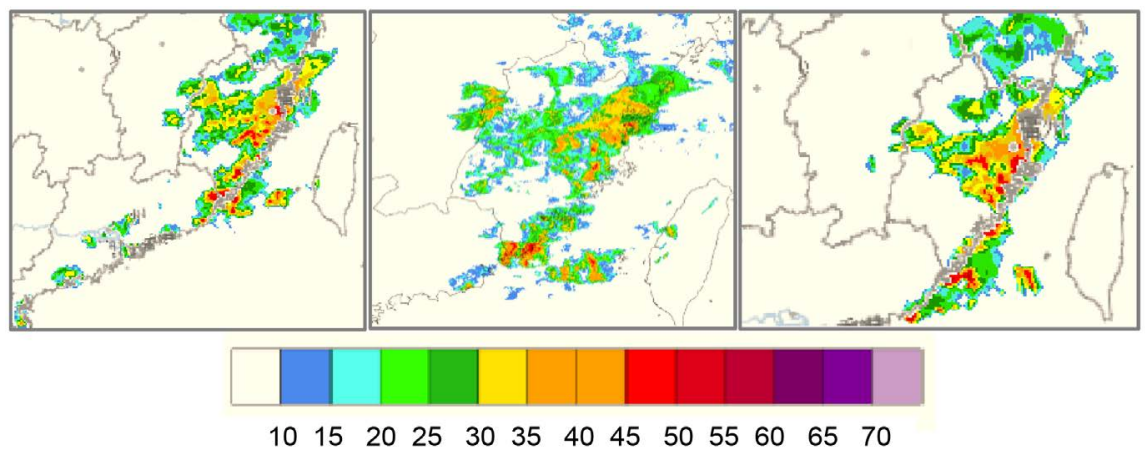

Figure 1. 19:00, April 11, 2017 (Beijing time). (a) Live echo; (b) Swan 10 hour forecast for 1 hour extrapolation echo; (c) Optical flow method 10 hour forecast for extrapolated echo after 1 hour. 


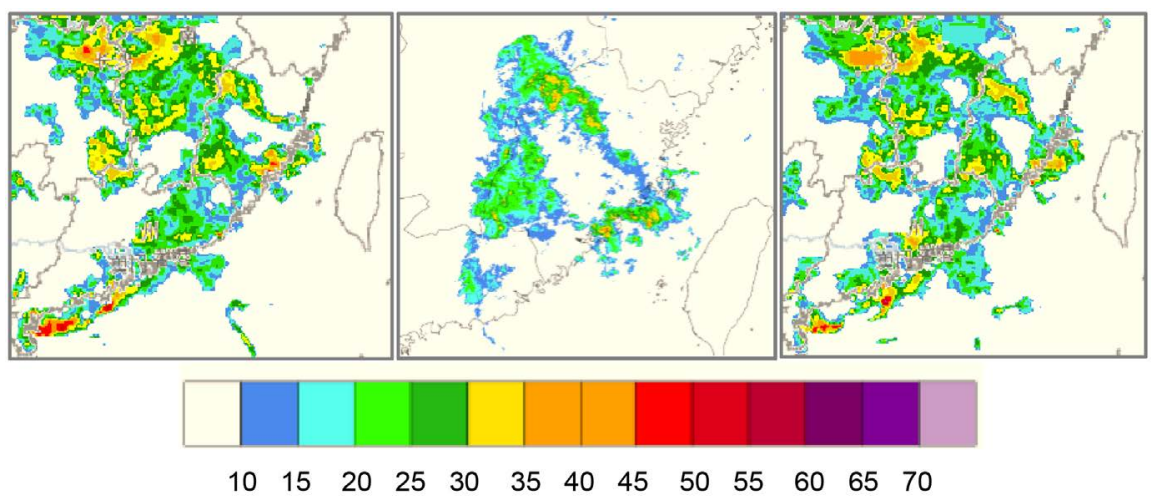

Figure 2. At 22 o'clock on April 24, 2017 (Beijing time). (a) Live echo; (b) Swan 21 hour forecast for 1 hour extrapolation echo; (c) Optical flow method 21 hour forecast for 1 hour extrapolation echo.

forecasted. It can be seen that for the convection, the optical flow method is also more effective than the SWAN method.

By combining the optical flow method and the Lagrangian extrapolation method, the radar echo is predicted and predicted. By comparison, it is found that the scheme is superior to the SWAN product and has obvious advantages in strength prediction. At the same time, the optical flow method is extrapolated and cross-correlated. There are limitations as well, that is, there is still a certain difficulty in short-term local consumption prediction, and it is necessary to introduce a model dynamic mechanism to influence the prediction scheme of improving echo cancellation. At present, the optical flow method is gradually used to extrapolate and forecast precipitation.

\subsection{Grid Fusion Technology}

\subsubsection{Grid Fusion Technology}

Combining the advantages of two fusion technologies, dynamic weighting method and trend evolution superposition method (Yang et al., 2010), a grid fusion technology is established. 1) Dynamic weight fusion method: The relative weights of radar QPF and numerical model output prediction values need to be adjusted with time. In the aging node, the radar QPF prediction value takes a larger weight, but with the extension of the forecasting time, the radar QPF The error increases, so the numerical forecast results are given a relatively large weight when the forecast period is long to prolong the timeliness and accuracy of the forecast. 2) Trend evolution superposition method: Calculate the quantitative trend of the convective system enhancement weakening of the numerical model prediction corresponding to the extrapolation time of each pixel point, and superimpose it with the radar QPF, so that the forecast result can better reflect the development trend of the convective system. In a certain area, find the development trend of the numerical model forecast rainfall in the extrapolation time corresponding to each grid point, quantify it, and then superimpose this quantification trend into the radar QPF forecast result. In the selected region, using the numerical model predicted from the actual t-time to predict the 
numerical prediction of the predicted time of $(t+\Delta t)$ and $(t+\Delta t-1)$, the development trend of the numerical model corresponding to the extrapolation time corresponding to each pixel point can be obtained:

$\operatorname{Rt}(t+\Delta t)=\operatorname{Rm}(t+\Delta t)-\operatorname{Rm}(t+\Delta t-1)$ where $\operatorname{Rt}(t+\Delta t)$ is the development trend of the numerical model corresponding to each lattice point, and $\operatorname{Rm}(t+\Delta t)$ is the time of the numerical model prediction $(t+\Delta t)$ The rainfall, $\operatorname{Rm}(t+\Delta t-1)$ is the rainfall at the time of $(t+\Delta t-1)$ predicted by the numerical model. Then the development trend of the numerical model prediction is superimposed with the interpolated radar QPF prediction to obtain the fusion value: $\mathrm{R}(t+\Delta t)=\operatorname{Rr}(t+\Delta t)+\operatorname{Rt}(t+\Delta t)$ where $\mathrm{R}(t+\Delta t)$ is the time after fusion $(t+\Delta t)$ The rainfall, $\operatorname{Rr}(t+\Delta t)$ is the radar QPF rainfall.

\subsubsection{Establishing a Grid Fusion Solution}

Based on the above-mentioned precipitation prediction error test statistical evaluation results (Table 1), it is determined that the radar QPF quantitative precipitation prediction error is smaller than the aging node of the model precipitation forecast, etc., using Doppler radar three-dimensional puzzle program, combined with Jianyang, Longyan, Xiamen, Changle, Sanming, Quanzhou 6 CINRAD/SA radar-based data, ground automatic station rainfall data and radar QPF precipitation products, using grid fusion technology, comprehensive error analysis results using error correction coefficient to determine the weight function, establishing measured rainfall, HRRR mode precipitation forecast, Radar QPF three-source fusion system correction scheme, output time resolution of 6 minutes, spatial resolution of $1 \mathrm{~km} \times 1 \mathrm{~km}$, time accumulation of 1 hour precipitation forecast products.

\subsection{Establish an Optimal Correction Plan}

Using the radar data optical flow method to extrapolate the minute scale, and cooperate with the rapid assimilation mode mesoscale numerical model HRRR of Fujian Province (the product as the environmental field, make full use of the advantages of both, and propose the dynamic weight fusion method to integrate the radar data with the numerical model product. Strong convective weather short-term nowcasting: The relative weight of the radar extrapolated QPF value and the numerical model's output forecast value needs to be adjusted with time. In a shorter forecasting time, the radar extrapolation method predicts a relatively

Table 1. Monthly and TS report rate for April-June 2017.

\begin{tabular}{cccc}
\hline Time & evaluation variable & SWAN & NOWCAST \\
\hline \multirow{2}{*}{ April 2017 } & 1 hour storm TS score & $16.3 \%$ & $16.6 \%$ \\
May 2017 & 1 hour storm TS miss rate & $79.9 \%$ & $55.0 \%$ \\
& 1 hour storm TS score & $16.8 \%$ & $16.6 \%$ \\
June 2017 & 1 hour storm TS miss rate & $80.0 \%$ & $62.1 \%$ \\
& 1 hour storm TS score & $16.6 \%$ & $16.4 \%$ \\
& 1 hour storm TS miss rate & $80.9 \%$ & $58.6 \%$
\end{tabular}


large weight. However, with the extension of the forecasting time, the error of the radar extrapolation forecast increases, so when the forecast period is long, the numerical forecast results are given a relatively large weight to prolong the timeliness and accuracy of the forecast. The weight of the numerical model forecast is calculated using the sinusoidal weight. Finally, based on the weights obtained, the radar extrapolation is combined with the numerical model prediction. The radar weight is $W_{r}(t)$, the mode weight is $W_{r}(t)$, and $t$ is time.

$$
\begin{aligned}
& W_{m}(t)=\sin ^{2}\left(-\frac{\pi}{3} t+\frac{7 \pi}{6}\right) \\
& W_{r}(t)=\cos ^{2}\left(-\frac{\pi}{3} t+\frac{7 \pi}{6}\right)
\end{aligned}
$$

The method of integrating rainfall is as follows:

$$
R(t)=W_{r}(t) R_{r}(t)+W_{m}(t) R_{m}(t)
$$

$R(t)$ is the amount of rainfall after the fusion, $R_{r}(t)$ is the rainfall obtained by the radar extrapolation forecast $R_{m}(t)$ is the rainfall predicted by the numerical model.

Combining various error analysis results, the error correction coefficient is used to determine the scheme, and based on the correction effect, the fusion parameters are continuously adjusted, and the preferred scheme is adopted to optimize the correction scheme. Furthermore, the forecasting products of precipitation rolling update based on measured dense data and short-term forecast are obtained. The system outputs objective precipitation forecast grid products and drop forecast products (contours, images, texts).

\section{Precipitation Rolling Update Revised Forecast Product Inspection and Evaluation}

After the real-time effect rolling inspection and evaluation of the precipitation rolling update correction products based on the measured dense data and the short-term forecasting is carried out, and the precipitation rolling update revised forecasting product is determined as the objective and optimal $1-3$ hour precipitation forecasting product.

\subsection{Sliding Up Update Revised Forecast Product Inspection and Evaluation}

The real-time effect rolling inspection and evaluation of the precipitation rolling correction products based on the measured dense data and the short-term forecasting is carried out, and the precipitation rolling revised forecasting product is determined as the objective and optimal 1 - 3 Hour precipitation forecasting product.

Extrapolation of the radar extrapolation results at 14:00 on 15 June 2017 (Figure 3(b)) and HRRR numerical model forecast results (Figure 3(b)) and 1 hour live 1 hour precipitation (Figure 3(a)) and condensed forecast rainfall 


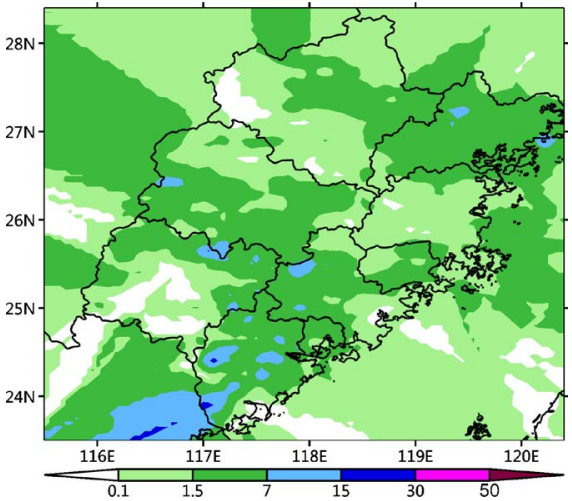

(a)

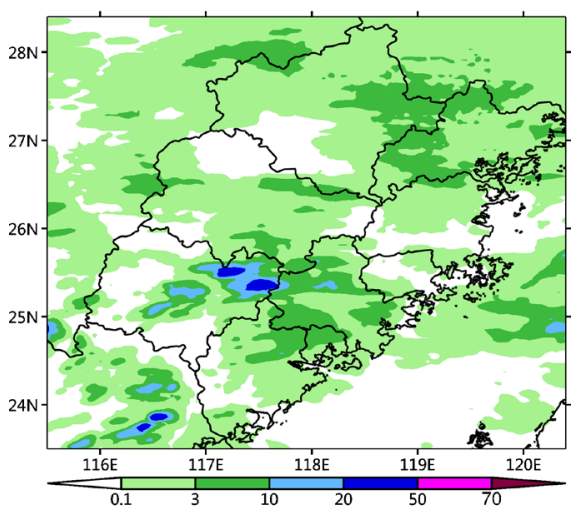

(c)

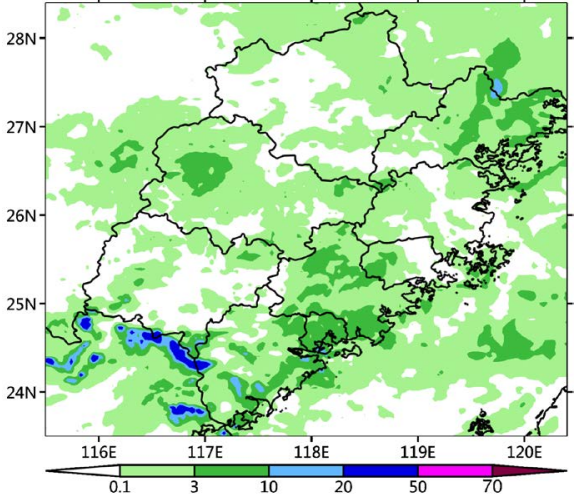

(b)

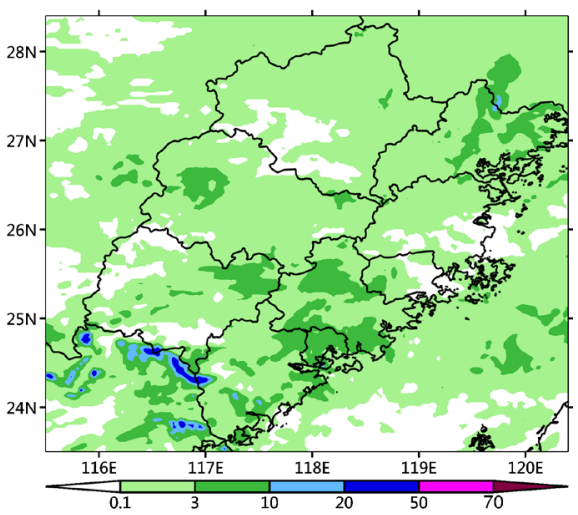

(d)

Figure 3. 15:00, June 15, 2017 (Beijing time). (a) Live 1 hour precipitation; (b) Optical flow prediction 1 hour QPF; (c) HRRR forecast 1 hour QPF; (d) Convergence forecast 1 hour QPF.

(Figure 3(d)). It can be seen from the comparison that the radar extrapolation forecast is basically accurate in the precipitation area in the southern part of the province, but the estimation of rainfall is not very accurate, and the change of the central rain area and the rainfall amount is not predicted. The estimation of rainfall in the central part of our province is generally too large, and the forecast of the center position of the convective monomer is deviated from the actual situation. According to Figure 3(d), the radar extrapolation and numerical model are combined to correct the rain area of the radar extrapolation forecast, and the rainfall is also corrected, reflecting the tendency of the convective system to weaken.

Extrapolation of the radar extrapolation results from 16:00 on June 15, 2017 to 17 o'clock (Figure 4(b)) and HRRR numerical model prediction results (Figure $4(c)$ ) and 1 hour live 1 hour precipitation (Figure 4(a)) and convergent forecast rainfall (Figure 4(d)) It can be seen that the live one-hour precipitation area is in the area of Longyan and Zhangzhou. The HRRR is reported to be above 50 $\mathrm{mm}$, but the position is eastward. The fusion forecast and radar extrapolation are more consistent, but the $50 \mathrm{~mm}$ range It is too small, but for the precipitation in Ganzhou, the fusion forecast is closer to the reality. 


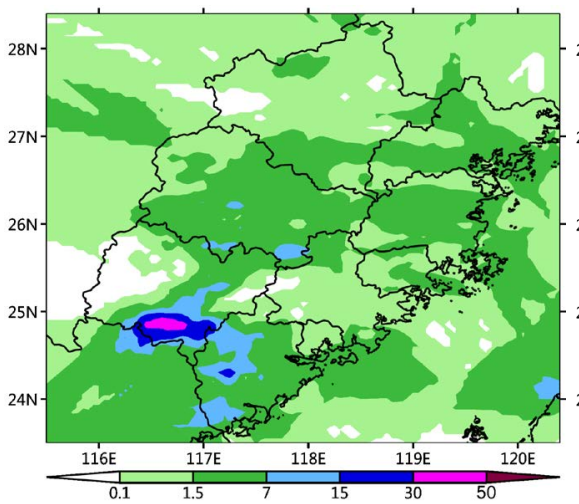

(a)

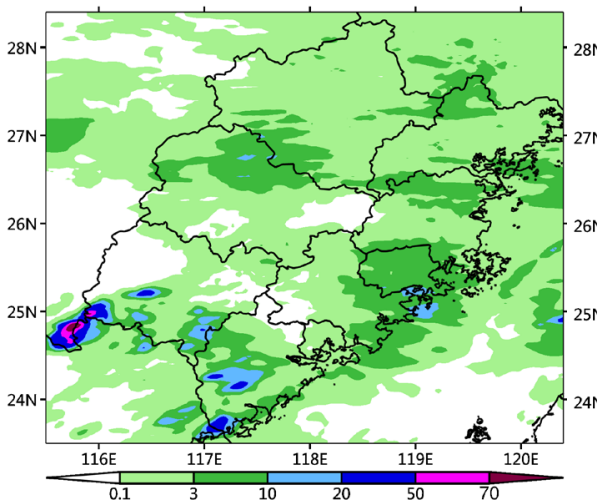

(c)

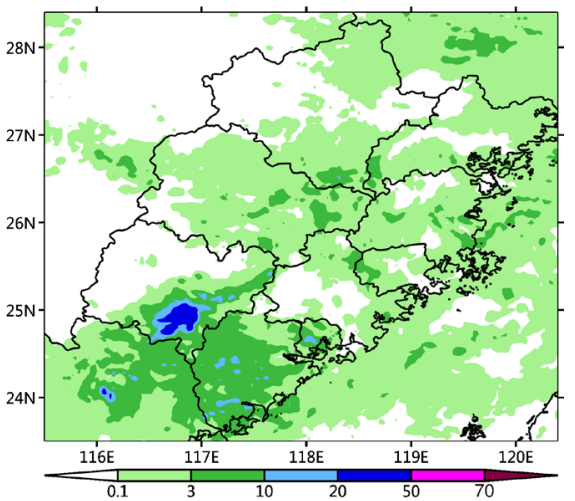

(b)

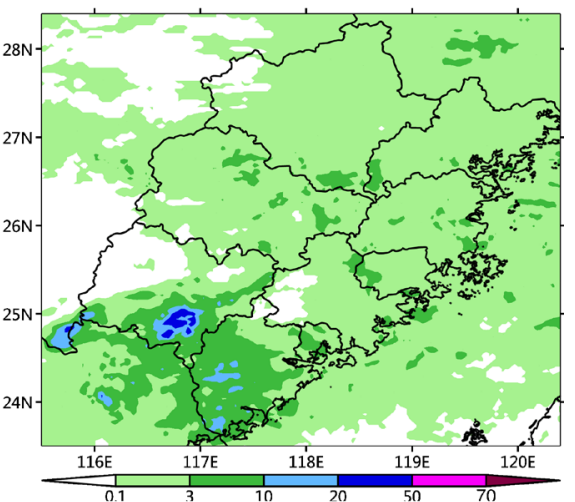

(d)

Figure 4. 17:00, June 15, 2017 (Beijing time). (a) Live 1 hour precipitation; (b) Optical flow prediction 1 hour QPF; (c) HRRR forecast 1 hour QPF; (d) Convergence forecast 1 hour QPF.

Extrapolation of the radar extrapolation forecast at 1700 hours on 15 June 2017 (Figure 5(b)) with the HRRR numerical model forecast (Figure 5(c)) and the 15-hour live 1 hour precipitation (Figure 5(a)) and the combined forecast rainfall (Figure 5(d)). It can be seen that the live one-hour precipitation area is in the area of Longyan and Zhangzhou. The HRRR is reported to be above 50 $\mathrm{mm}$, but the position is obviously large and the magnitude is also high. The fusion forecast and radar extrapolation More consistent, but the $10 \mathrm{~mm}$ range is closer to the live, the fusion forecast reflects the HRRR's heavy rain, but the extrapolation advantage reduces the scope of the heavy precipitation. Compared with the radar extrapolation and numerical model prediction, the predicted forecast results are closer to the actual situation, and the numerical model predicts the problem that the rain intensity is generally too large, and the deviation of the rain intensity center position is corrected.

\subsection{Hourly TS Score}

The following Figure 6 gives the various magnitudes of TS for the period of June $14-15,2017$. It can be seen that the fusion forecast is significantly better than the HRRR and extrapolation forecasts at various magnitudes below $7 \mathrm{~mm}$, indicating 


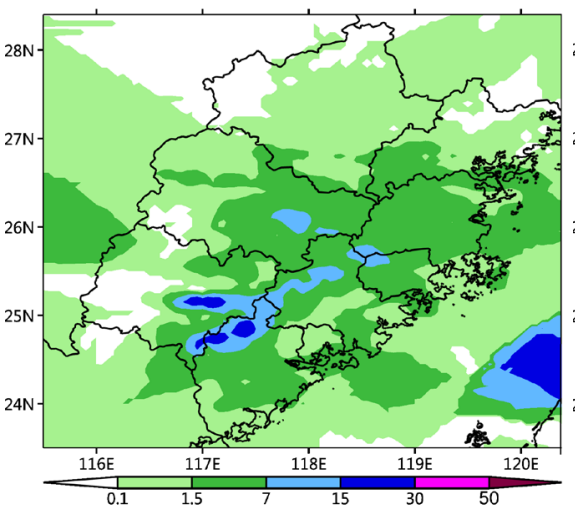

(a)

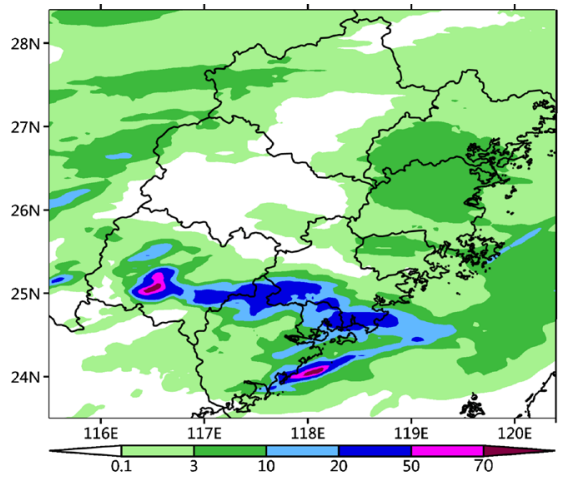

(c)

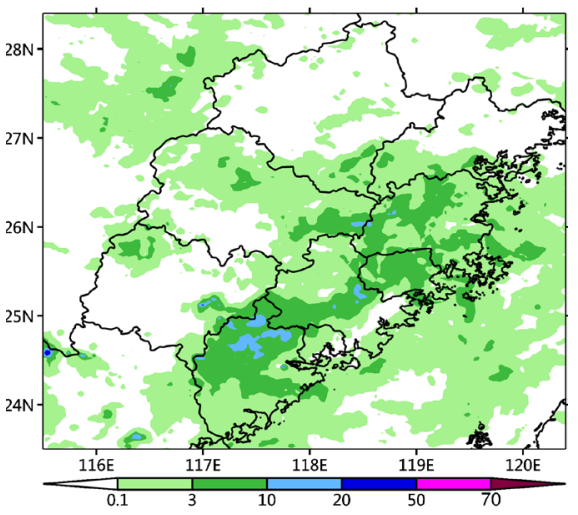

(b)

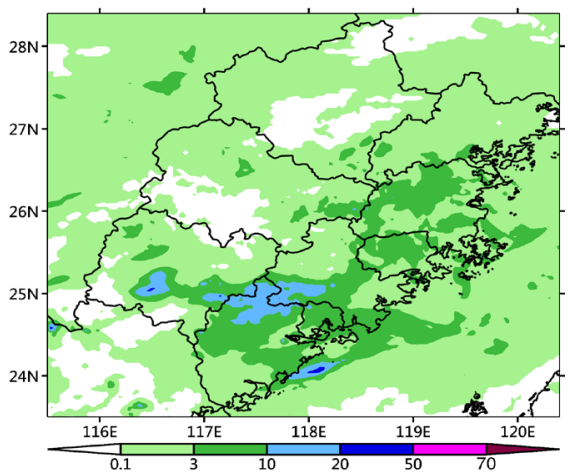

(d)

Figure 5. 19:00, June 15, 2017 (Beijing time). (a) Live 1 hour precipitation; (b) Optical flow prediction 1 hour QPF; (c) HRRR forecast 1 hour QPF; (d) Convergence forecast 1 hour QPF.

that the fusion forecast effectively reduces the forecast bias of weak precipitation. The forecast between 7 - $30 \mathrm{~mm}$ also maintains the optimal level of extrapolation and mode, combining the advantages of both, but the prediction effect of all members above $30 \mathrm{~mm}$ is not good, indicating that the fusion forecast is also limited by predictability. The process of pushing and mode cannot be reported, and the fusion cannot be predicted.

\subsection{HRRR Model Forecasting, Optical Flow Extrapolation Forecasting and Fusion Product Predictable Test Evaluation}

From the fusion product (blue line), WRF mode prediction (brown line) and optical flow method extrapolation forecast (light blue line) precipitation CSI with time curve (Figure 7) available: optical flow radar extrapolation forecast There is an advantage in $0-2$ hours, the fusion product is better than WRF mode prediction (brown line) and radar extrapolation forecast in $0-6$ hours, and over time, that is, over 6 hours, high resolution numerical prediction mode The advantages are becoming more and more apparent.

\section{Conclusion}

For the nowcasting around $0-2 \mathrm{~h}$, the fusion prediction effect is better than the 
mode prediction effect. The optical flow radar extrapolation forecast has an advantage in $0-2$ hours, and the fusion product performs better than the WRF mode prediction (brown line) and the radar extrapolation forecast in 0 - 6 hours. Over time, it is more than 6 hours. The advantages of high-resolution numerical

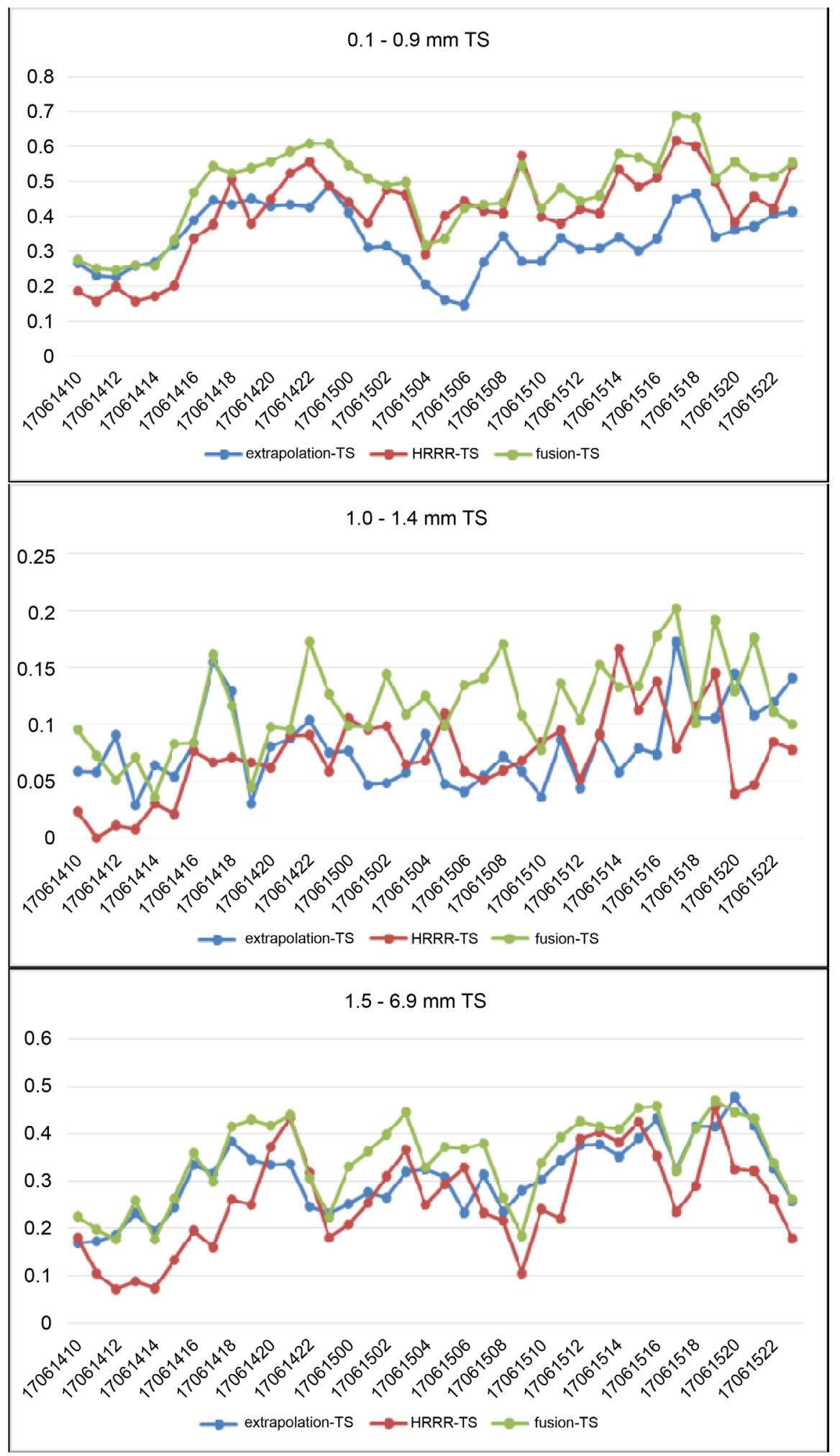




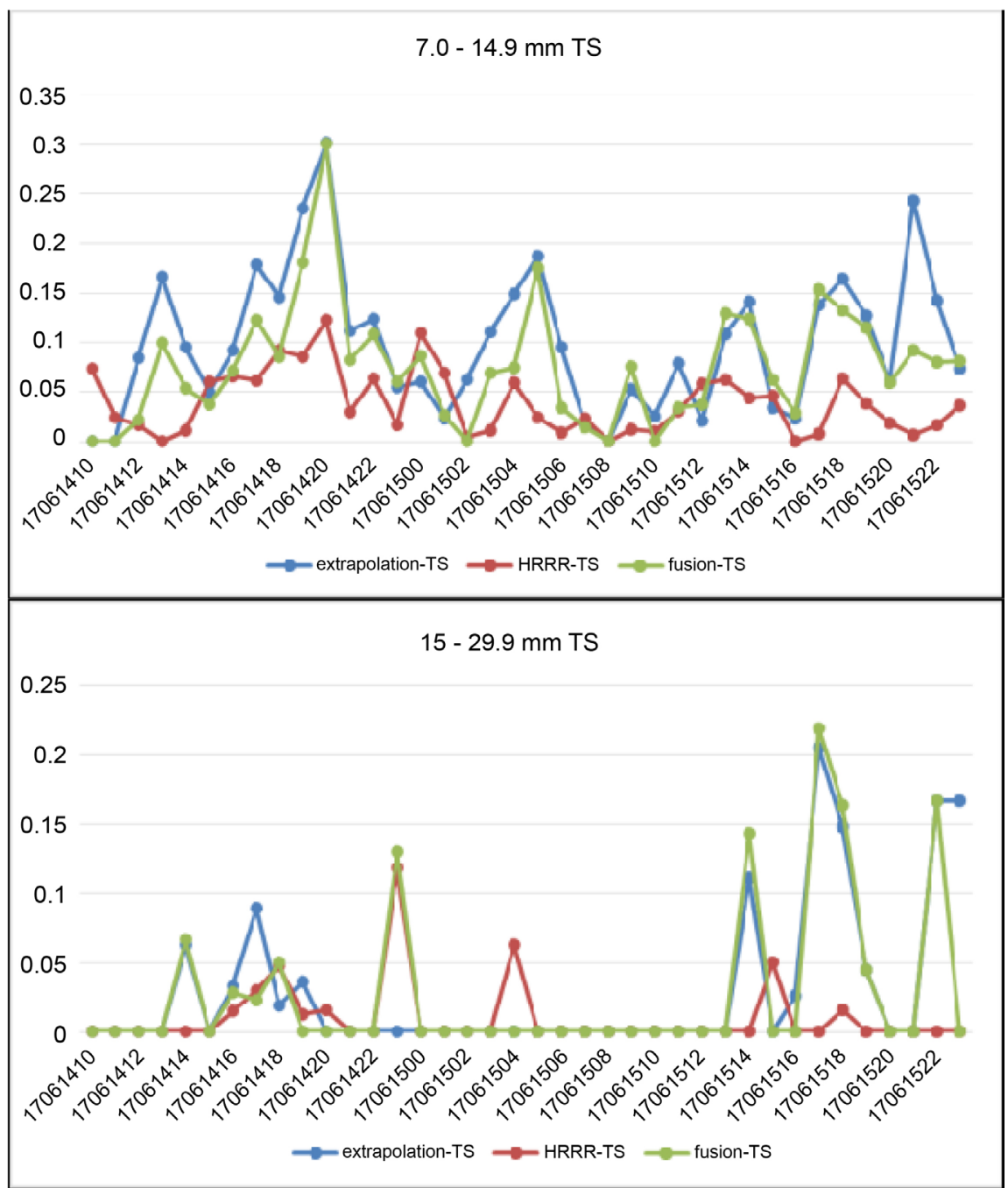

Figure 6. (a) $0.1-0.9 \mathrm{~mm}$; (b) $0.1-0.9 \mathrm{~mm}$; (c) 1.5 - $6.9 \mathrm{~mm}$; (d) $7.0-14.9 \mathrm{~mm}$; (e) 15 $29.9 \mathrm{~mm}$ extrapolation-TS, HRRR-TS and fusion-TS hourly distribution.

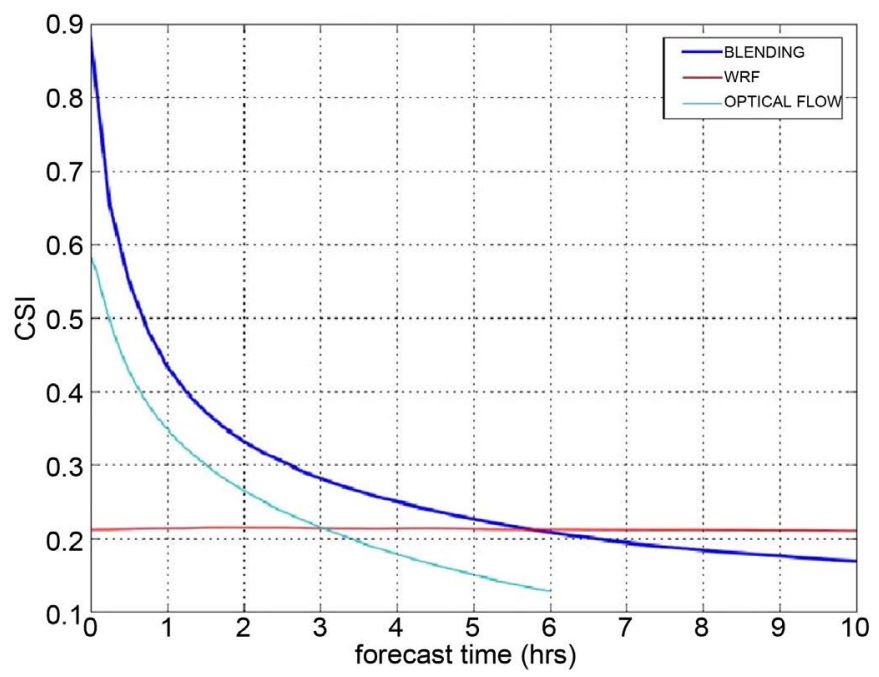

Figure 7. Convergence product (blue line), WRF forecast (brown line) and optical flow extrapolation forecast (light blue line) precipitation CSI versus time curve. 
prediction models are becoming more and more apparent.

\section{Discussion}

1) Using the optical flow method combined with the semi-Lagrangian tracking extrapolation algorithm (abbreviated as optical flow method) to extrapolate the precipitation forecast at the minute level: when the echo extrapolation forecasts, the COTREC cross-correlation method is changed to the optical flow method. The optical flow method is also of great reference value for the one-hour extrapolation forecast of a wide range of convective systems. The optical flow method improves the extrapolation effect of convective echoes and improves the prediction ability of radar echo movement. Combined with the semi-Lagrangian scheme, the echo is extrapolated, the rotation of the echo is maintained, and the echo prediction effect is improved.

2) Grid fusion technology is established: combining the advantages of two fusion technologies, dynamic weighting method and trend evolution superposition method, a grid fusion technology is established.

3) Establish an optimal correction plan: based on the real-time effect rolling test to evaluate the correction effect, continuously adjust the fusion parameters, and adopt the preferred scheme to make the correction scheme optimal. Furthermore, the forecasting products of precipitation rolling update based on measured dense data and short-term forecast are obtained. The combination of radar extrapolation and numerical model corrections corrected the rain area of the radar extrapolation forecast, and the rainfall was also corrected, reflecting the tendency of the convective system to weaken.

4) High-resolution precipitation rolling update of revised products improves the availability of model precipitation forecast, and helps to improve the accuracy and refinement level of short-term monitoring and early warning; fusion technology extends the extrapolation time.

\section{Acknowledgements}

This paper is sponsored by the Public Welfare Industry (Meteorological) Research Project (GYHY201506022), the Fujian Provincial Meteorological Bureau Open-end Fund Project (2016K01), and the Fujian Provincial Natural Science Foundation Project (2016J01182), China, and China Meteorological Administration Forecaster Special Project (CMAYBY2019-55).

\section{Conflicts of Interest}

The authors declare no conflicts of interest regarding the publication of this paper.

\section{References}

Awadallah, A., \& Awadallah, N. (2013). A Novel Approach for the Joint Use of Rainfall Monthly and Daily Ground Station Data with TRMM Data to Generate IDF Estimates 
in a Poorly Gauged Arid Region. Open Journal of Modern Hydrology, 3, 1-7. https://doi.org/10.4236/ojmh.2013.31001

Chen, M., Yu, X., Tan, X. et al. (2004). Development and Research Progress of Convective Weather Nowcasting Technology. Journal of Applied Meteorology, 15, 754-766.

Dong, Q. (2018). Calibration and Quantitative Forecast of Extreme Daily Precipitation Using the Extreme Forecast Index (EFI). Journal of Geoscience and Environment Protection, 6, 143-164. https://doi.org/10.4236/gep.2018.62010

Goerss, J. S., \& Jeffries, R. A. (1994). Assimilation of Synthetic Tropical Cyclone Observations into the Navy Operational Global Atmospheric Prediction System. Weather \& Forecasting, 9, 557-576. https://doi.org/10.1175/1520-0434(1994)009<0557:AOSTCO>2.0.CO;2

Hu, S., Sun, G., Zheng, Y. et al. (2011). The Characteristics of the Nowcasting System (SWAN) and Its Application in the Strong Convection Process in Guangzhou on May 7, 2010. Guangdong Meteorology, 33, 11-15.

Huang, W., Zhang, X., \& Wei, X. (2011). An Improved Contract Net Protocol with Multi-Agent for Reservoir Flood Control Dispatch. Journal of Water Resource and Protection, 3, 735-746. https://doi.org/10.4236/jwarp.2011.310084

Kissi, A., Abbey, G., Agboka, K., \& Egbendewe, A. (2015). Quantitative Assessment of Vulnerability to Flood Hazards in Downstream Area of Mono Basin, South-Eastern Togo: Yoto District. Journal of Geographic Information System, 7, 607-619. https://doi.org/10.4236/jgis.2015.76049

Long, Q., Liu, H., Gu, J. et al. (2014). Research on Fusion Method of Radar Data and Mesoscale Numerical Prediction and Its Application in Nowcasting. Meteorology, 40, 1248-1258.

Sakijege, T., Sartohadi, J., Marfai, M., Kassenga, G., \& Kasala, S. (2014). Government and Community Involvement in Environmental Protection and Flood Risk Management: Lessons from Keko Machungwa, Dar es Salaam, Tanzania. Journal of Environmental Protection, 5, 760-771. https://doi.org/10.4236/jep.2014.59078

Tanessong, R., Vondou, D., Igri, P., \& Kamga, F. (2017). Bayesian Processor of Output for Probabilistic Quantitative Precipitation Forecast over Central and West Africa. Atmospheric and Climate Sciences, 7, 263-286. https://doi.org/10.4236/acs.2017.73019

Yang, D., Shen, S., Shao, L. et al. (2010). Radar Data and Numerical Model Product Fusion Technology Research. Meteorology, 36, 53-60.

Zhang, X., Liu, J., Gao, Y., \& Yang, X. (2017). Study on Precipitation Forecast and Testing Methods of Numerical Forecast in Fuxin Area. Journal of Geoscience and Environment Protection, 5, 32-38. https://doi.org/10.4236/gep.2017.57004 